\title{
Risk of treatment related death (TRD) with adjuvant chemotherapy for breast cancer: A study in University Malaya Medical Centre (UMMC)
}

\author{
Chee Ee Phua ${ }^{1 *}$, Anita Zarina Bustam¹, Mastura Md Yusof ${ }^{1}$, Marniza Saad ${ }^{1}$, Cheng-Har Yip ${ }^{1}$, Nor Aishah Taib ${ }^{1}$ and Char Hong Ng ${ }^{1}$ \\ Correspondence: vince phua@yahoo.com \\ ${ }^{1}$ Clinical Oncology Unit, Faculty of Medicine, University of Malaya, Kuala Lumpur, Malaysia.
}

\begin{abstract}
Background: The risk of TRD with adjuvant chemotherapy for early breast cancer is unknown in Malaysia despite its widespread usage. This study aims to determine this rate in a large cohort of patients treated in UMMC.

Patients \& Methods: Patients who were treated with neoadjuvant or adjuvant chemotherapy for early breast cancer stages I, II or III from 2000-2007 in UMMC were identified from our UMMC Breast Cancer Registry. The TRD rate and 5 years overall survival (OS) were determined. TRD is defined as death occurring during or within 30 days of completing chemotherapy as a consequence of the chemotherapy treatment. OS was defined as death from any cause from the date of diagnosis to the date of death. OS was determined using the Kaplan-Meier method and differences between AJCC stages were compared by log-rank test.

Results: A total of 1317 were identified for analysis. The median age at diagnosis was 49 years with a range of 24 to 74 years. The rate of TRD was $0.1 \%$. The 5 years OS rate was $77.3 \%$ with a median follow up of 62 months. The 5 years OS according to AJCC stage were $90.7 \%$ for stage I, $83.9 \%$ for stage II and $62.2 \%$ for stage III disease. The commonest chemotherapy regimen used was the FEC (5-Fluorouracil, Epirubicin, Cyclophosphamide) regimen accounting for approximately $90 \%$ of the cases.
\end{abstract}

Conclusion: Adjuvant chemotherapy for early breast cancer with the FEC regimen is safe with a TRD rate of $0.1 \%$ in our centre.

Keywords: treatment related death (TRD), breast cancer, adjuvant chemotherapy

\section{Background}

The role of chemotherapy in cancer treatment is highly misunderstood not just in the public domain but also amongst medical practitioners. The fact that chemotherapy only plays a minor role as a definitive modality of cancer treatment is not well understood and it is mainly limited to haematological malignancies and germ cell tumours. Chemotherapy's role in the treatment of solid tumours is mainly confined to neoadjuvant, adjuvant and palliative settings. As such, it is of utmost importance to be cognizant to the rate of TRDs with chemotherapy other than being aware of its potential benefits when used in these settings. Informing patients that this risk is negligible or less than one percent which is the figure often quoted by clinicians may be inadequate as this gives the impression that it is extremely rare. Moreover less than 1\% can mean anywhere between 1 in 1001, 1 in 10000, 1 in 100000, 1 in 1000000 and so forth. Patients deserve to fully understand the real risk of TRDs and equally important is the need for clinicians to realize that TRDs are much commoner than perceived. There are various definitions of TRD used in the literature. For the purpose of this study the TRD was defined as deaths that occurred less than or equal to 30 days after the last cycle of chemotherapy, death of which was due to the chemotherapy itself. This definition was chosen as it was commonly used in many reported phase 3 trials and it is the least ambiguous amongst all definitions encountered in the literature. One major drawback is the fact that it does not take into account deaths that may be related to the long term side effects of chemotherapy for example deaths due to cardiac events which is especially relevant for anthracycline drugs commonly used for breast cancer treatment. Having said that, it is very difficult to prove that these deaths are directly due to the effect of chemotherapy alone as there are frequently many other confounding factors.

Breast cancer is the commonest cancer treated with adjuvant chemotherapy in Malaysia. A commonly used regimen is the FAC regime (5 Fluorouracil, Adriamycin, Cyclophosphamide). In a trial involving 1491 patients in node positive breast cancer patients post definitive surgery, 745 patients received adjuvant TAC (Docetaxel, Adriamycin, Cyclophosphamide) and 746 patients received FAC. The TRDs for the TAC group and FAC group was similar at $0.3 \%$. [1]. The commonest regime used in our center is the FEC regime (5 Fluorouracil, Epirubicin, Cyclophosphamide). A pivotal phase 3 study, PACS 01 trial compared the FEC regime for 6 cycles with the FEC-D regime (FEC3-Docetaxel3) involving 996 patients in the FEC arm and 1003 patients in the FEC-D arm. Although significant high rates of febrile neutropaenia at $8.4 \%$ and $11.2 \%$ were reported in the FEC and FEC-D arms respectively, there were no early TRDs. However, there was one delayed cardiac death in each 
group [2]. In another trial which compared two different FEC regimens; FEC50 (epirubicin $50 \mathrm{mg} / \mathrm{m} 2$ ) and FEC100 (epirubicin $100 \mathrm{mg} / \mathrm{m} 2$ ), there was also no early TRD in the 565 patients recruited for this trial [3]. A subsequent update of this trial showed an absolute 10 years overall survival (OS) benefit of $4.8 \%$ in favour of the FEC100 regimen and this advantage was not offset by long term toxicity. The authors concluded that the FEC100 regime is associated with an acceptably low risk of long term cardiac toxicity and second malignancy with a rate of $1.1 \%$ and $8.3 \%$ respectively [4]. Although the FEC and FEC-D regimens appear to be safe at least with regards to early TRD, this was shown in highly controlled phase 3 trials. A retrospective study in Ontario, Canada which looked at the safety profile of the FEC-D regimen administered to 671 patients revealed a worrying $22.7 \%$ incidence rate of febrile neutropaenia and there were 3 TRDs (0.4\%). The authors concluded that based on the accepted threshold level of $20 \%$, primary haematopoietic growth factor prophylaxis should be adopted in routine clinical practice when using adjuvant FEC-D regimen [5]. This strongly suggests that actual observational phase 4 and population based studies are of utmost importance to ensure the safe translation of evidence based medicine into clinical practice for the general population. In another study evaluating sequential docetaxel as adjuvant chemotherapy for breast cancer (TACT), the TRD rate was $0.3 \%$ for the FEC-D regimen while the control arm using 4 cycles of epirubicin $100 \mathrm{mg} / \mathrm{m}^{2}$ followed by CMF regimen for another 4 cycles had a TRD of $0.1 \%$ while the control arm using FEC for 8 cycles had no TRD [6]. The US Oncology Research group reported a TRD of $0.4 \%$ with the TC regimen comprising of 4 cycles of Docetaxel and Cyclophosphamide and there was no TRD in the control arm using the AC regimen for 4 cycles (adriamycin and cyclophosphamide) in its phase 3 trial involving 1016 patients [7]. Another commonly used regimen is the $A C x 4$ followed by Paclitaxelx4 regimen. This regimen was compared to the $A C x 4$ regimen in the NSABP- 28 trial for node-positive breast cancer in the adjuvant setting. The TRD rates were $0.1 \%$ for the $\mathrm{AC} \times 4$ followed by Paclitaxelx 4 and $0.3 \%$ for the $\mathrm{ACx} 4$ regimen [8]. Above are the available data on TRDs with the commonest adjuvant chemotherapy used in this part of the world.

\section{Methods}

The data from the University Malaya Medical Centre (UMMC) Breast Cancer Registry, which was started in 1993 and where data on basic demography, clinical and pathological tumour profile, treatment details and survival was prospectively collected, was used for this study. We reviewed all patients prescribed with neoadjuvant or adjuvant chemotherapy for early-stage breast cancer in our centre from $1^{\text {st }}$ January 2000 till $31^{\text {st }}$ December 2007. Information collected included patient demographics, histopathological features, treatment received including the different chemotherapy regimens and presence of early TRD. The aim of this study is to establish the early TRD rate with adjuvant chemotherapy for early breast cancer in our centre. TRD is defined as death occurring during or within 30 days of completing chemotherapy as a consequence of the chemotherapy treatment. OS was defined as death from any cause from the date of diagnosis to the date of death. Statistical analysis was performed using the SPSS version 18.0 software. Survival probabilities were estimated using the Kaplan-Meier method and differences in survival compared using log-rank test.

\section{Results}

Between $1^{\text {st }}$ January 2000 and $31^{\text {st }}$ December 2007, 2536 patients with newly diagnosed breast cancer were treated in UMMC. A total of 1317 of these patients received neoadjuvant or adjuvant chemotherapy for either AJCC stage I, II or III diseases. Analysis was therefore performed on these 1317 patients. Baseline characteristics including clinicopathological and treatment modalities utilized are summarized in Table 1. The mean age of patients in this study was 49 years (range 24 to 74 years). The mean size of the primary tumour was $4.0 \mathrm{~cm}$. The commonest neoadjuvant/adjuvant chemotherapy regimen used was the FEC75 (epirubicin $75 \mathrm{mg} / \mathrm{m}^{2}$ ) accounting for $87.9 \%$ of patients. This was followed by FEC-D, CMF (Cyclophosphamide, Methotrexate, 5-Fluorouracil), ACx4Paclitaxelx4 (Adriamycin, Cyclophosphamide, Paclitaxel), FEC(100), TAC, Docetaxel alone, FAC and finally AC regimen in descending order of frequency.

The main result of this study showed a TRD rate of $0.1 \%$ (1/1317). A consort table is shown in Table 2. The death occurred within 30 days of last dose of chemotherapy and it occurred after cycle 6 of chemotherapy. The cause of death was septicaemia. This patient had the FEC75 (epirubicin 75mg/ $\mathrm{m}^{2}$ ) chemotherapy regimen.

At a median follow up of 62 month, the 5 years OS rate was $77.3 \%$. The 5 years OS according to AJCC stage were $90.7 \%$ for stage I, $83.9 \%$ for stage II and $62.2 \%$ for stage III disease. The difference demonstrated in OS according to AJCC stage was statistically significant with a p-value of less than 0.000 as illustrated in Figure 1.

\section{Discussion}

The main finding of this study was the low TRD rate of $0.1 \%$, which is very reassuring for clinicians. As this study involved a large number of patients in a normal clinical situation i.e. not in a clinical trial setting this rate can be deemed to be an accurate reflection of the risk of TRD faced by patients treated with neoadjuvant/adjuvant chemotherapy for early breast cancer patients treated in the Klang Valley region in Malaysia. However, this TRD rate is only applicable to the FEC regimen as about $90 \%$ of the patients in this study were treated with this regimen. The finding is also consistent with the TRD rates reported in randomized control trials using adjuvant FEC regimens $[2-4,6]$. The role of adjuvant chemotherapy is firmly established in the treatment of early breast cancer. 
Phua et al. Journal of Cancer Therapeutics \& Research 2012,

http://www.hoajonline.com/journals/pdf/2049-7962-1-13.pdf

Table 1: The cohort of 1317 patients

\begin{tabular}{|c|c|c|}
\hline Items & $\begin{array}{c}\text { No.of } \\
\text { patients }\end{array}$ & $\%$ \\
\hline \multicolumn{3}{|l|}{ Age } \\
\hline Up to 50 years & 727 & 55.3 \\
\hline $51-69$ years & 578 & 44 \\
\hline 70 years and above & 12 & 0.7 \\
\hline \multicolumn{3}{|l|}{ Gender } \\
\hline Female & 1317 & 100 \\
\hline \multicolumn{3}{|l|}{ Race } \\
\hline Malay & 272 & 20.7 \\
\hline Chinese & 860 & 65.3 \\
\hline Indian & 172 & 13.1 \\
\hline Others & 12 & 0.9 \\
\hline \multicolumn{3}{|l|}{ Tumour Stage } \\
\hline TI & 350 & 26.7 \\
\hline $\mathrm{T} 2$ & 657 & 49.9 \\
\hline $\mathrm{T} 3$ & 152 & 11.6 \\
\hline $\mathrm{T} 4$ & 154 & 11.8 \\
\hline \multicolumn{3}{|l|}{ Nodal Stage } \\
\hline No & 566 & 42.9 \\
\hline N1 & 721 & 54.7 \\
\hline $\mathrm{N} 2$ & 20 & 1.6 \\
\hline N3 & 10 & 0.8 \\
\hline \multicolumn{3}{|l|}{ AJCC stage } \\
\hline I & 221 & 16.8 \\
\hline II & 627 & 47.6 \\
\hline III & 469 & 35.6 \\
\hline \multicolumn{3}{|l|}{ ER Status } \\
\hline Positive & 641 & 48.7 \\
\hline Negative & 638 & 48.4 \\
\hline Unknown & 38 & 2.9 \\
\hline \multicolumn{3}{|l|}{ HER-2 Status } \\
\hline Positive & 467 & 35.5 \\
\hline Negative & 672 & 51 \\
\hline Equivocal & 178 & 13.5 \\
\hline \multicolumn{3}{|l|}{ Chemotherapy } \\
\hline Neoadjuvant & 131 & 9.9 \\
\hline Adjuvant & 1186 & 90.1 \\
\hline \multicolumn{3}{|c|}{ Adjuvant Radiotherapy } \\
\hline Yes & 946 & 71.8 \\
\hline No & 371 & 28.2 \\
\hline \multicolumn{3}{|c|}{ Chemotherapy Regime } \\
\hline $\operatorname{FEC}(75)$ & 1158 & 87.9 \\
\hline $\operatorname{FEC}(100)$ & 18 & 1.4 \\
\hline FEC-D & 50 & 3.8 \\
\hline FAC & 7 & 0.5 \\
\hline $\mathrm{ACx} 4$ & 5 & 0.4 \\
\hline ACx4-Paclitaxelx4 & 28 & 2.1 \\
\hline TAC & 13 & 1 \\
\hline $\mathrm{CMF}$ & 30 & 2.3 \\
\hline Docetaxel alone & 8 & 0.6 \\
\hline
\end{tabular}

Table 2: Flowchart for treatment related death

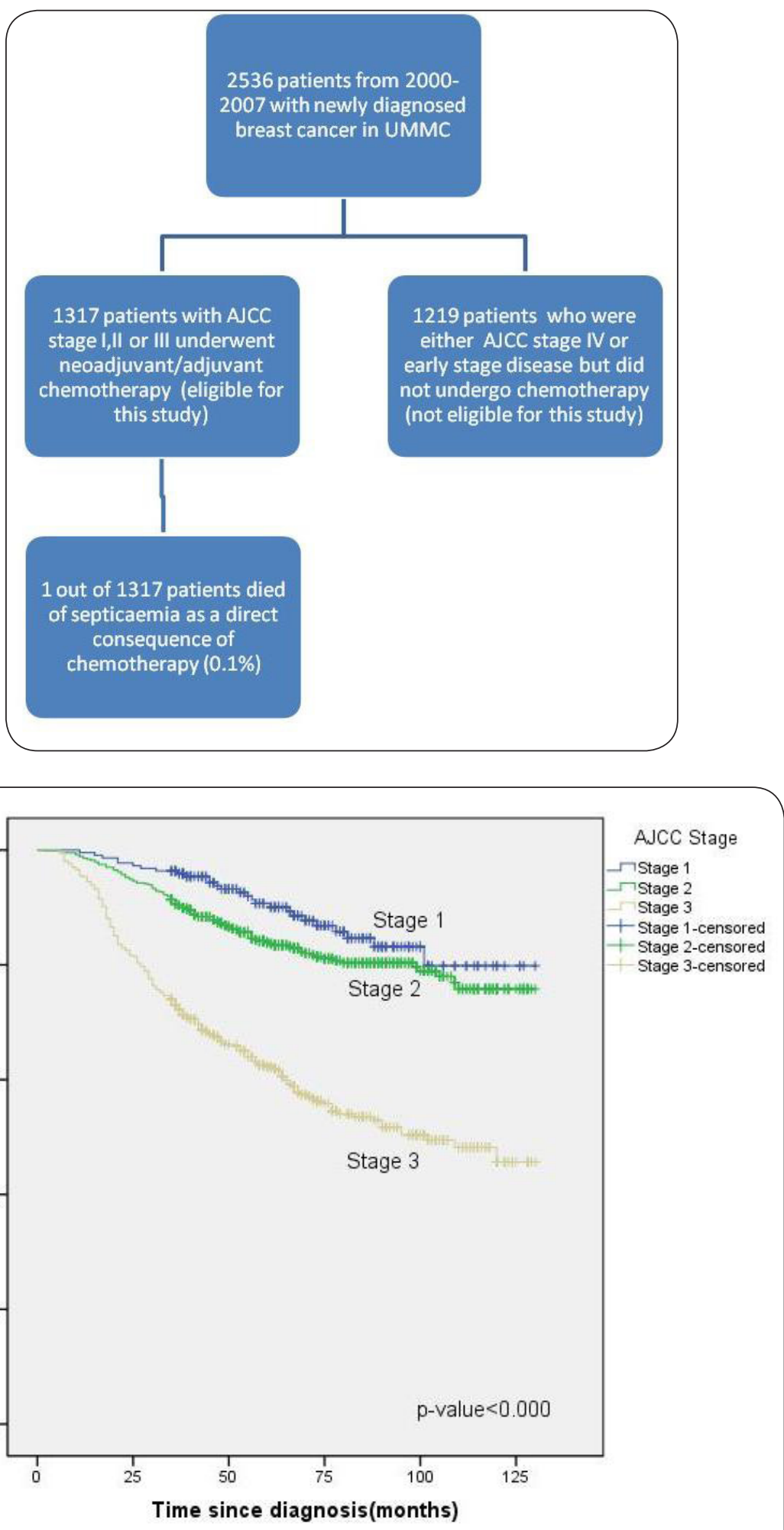

Figure 1. kaplan-meier estimates of overall survival according to ajcc stage 
The largest body of evidence comes from The Early Breast Cancer Triallists' Collaborative Group (EBCTCG) which involve collaborative meta-analyses undertaken on 194 unconfounded randomized trials of adjuvant chemotherapy or hormonal therapy that began by 1995 . The latest EBCTCG overview found a marked absolute reduction of 15 years breast cancer mortality rate with the use of adjuvant chemotherapy. It estimated an absolute reduction of $5 \%, 9 \%$ and $15 \%$ in breast cancer mortality at 15 years for patients younger than 50 years old with low, intermediate and high risk respectively. For patients who were 50-69 years old, the absolute reduction were $2 \%$, $4 \%$ and $7 \%$ with low, intermediate and high risk respectively [9]. With the availability of this important piece of data, clinicians in our centre can now confidently discuss the pros and cons of adjuvant chemotherapy with their patients. It is of utmost importance that clinicians and patients are cognizant to the fact that TRDs do occur and in the adjuvant setting treatment is given to asymptomatic patients who do not exhibit any evidence of disease clinically, biochemically or radiologically. TRDs in this setting can only be viewed as a devastating event from a medical point of view and all efforts must be made to ensure it remains as low as possible. This very low rate of $0.1 \%$ can be deemed as acceptable when compared to the potential survival benefit alluded to above. However, we must be careful not to extrapolate this rate for other treatment centers in Malaysia as UMMC is a tertiary medical centre treating on average a total of 300 newly diagnosed breast cancer patients per year. The rate of TRDs will be different in different centers especially in peripheral hospitals that only occasionally administer adjuvant chemotherapy.

Another point to note from this study is that about $10 \%$ of patients in this study received chemotherapy in the neoadjuvant setting. These patients did not have metastatic disease during their initial staging investigations. They were deliberately included for the purpose of this trial as the main aim of chemotherapy in this setting is for improvement of OS besides downstaging of disease to facilitate subsequent surgery. The survival benefit derived from either neo-adjuvant or adjuvant chemotherapy has been shown to be equivalent. This was clearly demonstrated in the meta-analysis comparing neoadjuvant versus adjuvant systemic treatment in breast cancer in an article published in 2005. A total of 9 trials involving 3861 patients were analyzed in this meta-analysis and it was found that there was no difference in the death rates between the 2 arms with a relative risk for death of $1.00(95 \% \mathrm{Cl}=0.90$ to 1.12) [10]. As such the TRD rate is relevant for both neoadjuvant and adjuvant chemotherapy when balancing the risk of death and the potential benefit of increased survival when employing systemic chemotherapy for early breast cancer.

This study also showed a 5 years overall survival rate of $90.7 \%, 83.9 \%$ and $62.2 \%$ for AJCC stages I, II and III respectively. These figures are comparable to the data reported by a Canadian group on 2192 breast cancer patients diagnosed between 1994-1997 in Ottawa, Canada. The reported figures in the Canadian trial were $96.0 \%, 85.6 \%$ and $59.2 \%$ for stages I, II and III respectively [11]. While our results for stage I and II are respectable, the 5 years overall survival rate for stage III disease remains low. Moreover, about a third of the patients in this study were diagnosed with stage III disease. It remains to be seen if the newer generation of adjuvant chemotherapy will impact on the survival for this group of patients. In fact the commonest regimen employed in our department at the moment is the FEC-D and the TC (Docetaxel, Cyclophosphamide) regimens besides the FEC(100) regimen. This is in view of the findings of a comprehensive systematic review by the Cochrane group on taxanes for adjuvant treatment of early breast cancer published in 2010. It found a hazard ratio for death of $0.81(95 \% \mathrm{Cl} 0.75$ to 0.88 , $\mathrm{P}<0.00001)$ favouring taxane containing regimens [12]. As taxane is being employed much more commonly now, another study looking at the rate of TRD with taxane containing regimens is urgently needed in our centre.

\section{Competing interests}

The Authors declare that they have no competing interests.

\section{Authors' contribution}

All authors contributed equally.

\section{Acknowledgements}

This study was supported by a HIR Grant UM.C/HIR/

MOHE/06 from the Ministry of Higher Education, Malaysia.

\section{Publication history}

Editor: Dmity Pshezhetskiy, Imperial College London, UK. EIC: G.J. Peters, VU University Medical Center, Netherlands. Received: 16-May-2012 Revised: 01-June-2012

Accepted: 06-June-2012 Published: 12-July-2012

\section{References}

1. Martin M, Pienkowski T, Mackey J, Pawlicki M, Guastalla JP, Weaver C, et al.: Adjuvant docetaxel for node-positive breast cancer. $N$ Engl J Med 2005; 352;(22.);2302-13. | Article | PubMed

2. Roche H, Fumoleau P, Spielmann M, Canon JL, Delozier T, Serin D, et al.: Sequential adjuvant epirubicin-based and docetaxel chemotherapy for node-positive breast cancer patients: the FNCLCC PACS 01 Trial. J Clin Oncol 2006; 24;(36.);5664-71. I Article I PubMed

3. French Adjuvant Study Group. Benefit of a high-dose epirubicin regimen in adjuvant chemotherapy for node-positive breast cancer patients with poor prognostic factors: 5-year follow-up results of French Adjuvant Study Group 05 randomized trial. J Clin Oncol 2001; 19;(3.);602-11. | Article | PubMed

4. Bonneterre J, Roche $\mathrm{H}$, Kerbrat $\mathrm{P}$, Bremond A, Fumoleau $\mathrm{P}$, Namer $\mathrm{M}$, et al.: Epirubicin increases long-term survival in adjuvant chemotherapy of patients with poor-prognosis, node-positive, early breast cancer: 10-year follow-up results of the French Adjuvant Study Group 05 randomized trial. J Clin Oncol 2005; 23;(12.);2686-93. | Article | PubMed

5. Madarnas Y, Dent SF, Husain SF, Robinson A, Alkhayyat S, Hopman WM, et al.: Real-world experience with adjuvant fec-d chemotherapy in four Ontario regional cancer centres. Curr Oncol 2011; 18;(3.);119-25. | Article | PubMed Abstract | PubMed Full Text

6. Ellis $\mathrm{P}$, Barrett-Lee $\mathrm{P}$, Johnson L, Cameron D, Wardley A, O'Reilly S, et al.: Sequential docetaxel as adjuvant chemotherapy for early breast cancer (TACT): an open-label, phase III, randomised controlled trial. 
Lancet 2009; 373;(9676.);1681-92. | Article | PubMed Abstract | PubMed Full Text

7. Jones S, Holmes FA, O'Shaughnessy J, Blum JL, Vukelja SJ, McIntyre KJ, et al.: Docetaxel With Cyclophosphamide Is Associated With an Overall Survival Benefit Compared With Doxorubicin and Cyclophosphamide: 7-Year Follow-Up of US Oncology Research Trial 9735. J Clin Oncol 2009; 27;(8.);1177-83. | Article | PubMed

8. Mamounas EP, Bryant J, Lembersky B, Fehrenbacher L, Sedlacek SM, Fisher B, et al.: Paclitaxel after doxorubicin plus cyclophosphamide as adjuvant chemotherapy for node-positive breast cancer: results from NSABP B-28. J Clin Oncol 2005; 23;(16.);3686-96. | Article I PubMed

9. Effects of chemotherapy and hormonal therapy for early breast cancer on recurrence and 15-year survival: an overview of the randomised trials. Lancet 2005; 365;(9472.);1687-717. | Article I PubMed

10. Mauri D, Pavlidis N, loannidis JP: Neoadjuvant versus adjuvant systemic treatment in breast cancer: a meta-analysis. J Nat/ Cancer Inst 2005; 97;(3.);188-94. | Article | PubMed

11. Ugnat AM, Xie L, Morriss J, Semenciw R, Mao Y: Survival of women with breast cancer in Ottawa, Canada: variation with age, stage, histology, grade and treatment. Br J Cancer 2004; 90;(6.);1138-43. | Article | PubMed Abstract | PubMed Full Text

12. Ferguson T, Wilcken N, Vagg R, et al. Taxanes for adjuvant treatment of early breast cancer (Review). The Cochrane Cochrane Library 2010, Issue 10.

\section{Citation:}

Phua CE, Bustam AZ, Yusof MM, Saad M, Yip CH, Taib $\mathrm{NA}$ and $\mathrm{Ng} \mathrm{CH}$ : Risk of treatment related death (TRD) with adjuvant chemotherapy for breast cancer: A study in University Malaya Medical Centre (UMMC). journal of Cancer Therapeutics and Research 2012, 1:13.

http://dx.doi.org/10.7243/2049-7962-1-13 